\title{
COEFFICIENT ESTIMATES FOR A CLASS OF ANALYTIC BI-UNIVALENT FUNCTIONS RELATED TO PSEUDO-STARLIKE FUNCTIONS
}

\author{
SERAP BULUT \\ Received 13 October, 2016
}

\begin{abstract}
In this paper we introduce and investigate an interesting subclass $\mathscr{L} \mathscr{B}_{\Sigma}^{h, p}(\lambda)$ of analytic and bi-univalent functions in the open unit disk $\mathbb{U}$. For functions belonging to the class $\mathscr{L} \mathcal{B}_{\Sigma}^{h, p}(\lambda)$, we obtain estimates on the first two Taylor-Maclaurin coefficients $a_{2}$ and $a_{3}$. The results presented in this paper would generalize and improve some recent work of Joshi et al. [5].

2010 Mathematics Subject Classification: $30 \mathrm{C} 45$
\end{abstract}

Keywords: analytic functions, univalent functions, bi-univalent functions, coefficient estimates, pseudo-starlike functions

\section{INTRODUCTION}

Let $\mathcal{A}$ denote the class of all functions of the form

$$
f(z)=z+\sum_{k=2}^{\infty} a_{k} z^{k}
$$

which are analytic in the open unit disk $\mathbb{U}=\{z: z \in \mathbb{C}$ and $|z|<1\}$. We also denote by $\&$ the class of all functions in the normalized analytic function class $\mathcal{A}$ which are univalent in $\mathbb{U}$.

Since univalent functions are one-to-one, they are invertible and the inverse functions need not be defined on the entire unit disk $\mathbb{U}$. In fact, the Koebe one-quarter theorem [4] ensures that the image of $\mathbb{U}$ under every univalent function $f \in \delta$ contains a disk of radius $1 / 4$. Thus every function $f \in \mathcal{A}$ has an inverse $f^{-1}$, which is defined by

$$
f^{-1}(f(z))=z \quad(z \in \mathbb{U})
$$

and

$$
f\left(f^{-1}(w)\right)=w \quad\left(|w|<r_{0}(f) ; r_{0}(f) \geq \frac{1}{4}\right) .
$$


In fact, the inverse function $f^{-1}$ is given by

$$
f^{-1}(w)=w-a_{2} w^{2}+\left(2 a_{2}^{2}-a_{3}\right) w^{3}-\left(5 a_{2}^{3}-5 a_{2} a_{3}+a_{4}\right) w^{4}+\cdots .
$$

A function $f \in \mathcal{A}$ is said to be bi-univalent in $\mathbb{U}$ if both $f$ and $f^{-1}$ are univalent in $\mathbb{U}$, in the sense that $f^{-1}$ has a univalent analytic continuation to $\mathbb{U}$. Let $\Sigma$ denote the class of bi-univalent functions in $\mathbb{U}$ given by (1.1). For a brief history and interesting examples of functions in the class $\Sigma$, see [6] (see also [2]). In fact, the aforecited work of Srivastava et al. [6] essentially revived the investigation of various subclasses of the bi-univalent function class $\Sigma$ in recent years; it was followed by such works as those by $\mathrm{Xu}$ et al. $[7,8]$.

Recently, Babalola [1] defined the class $\mathscr{L}_{\lambda}(\beta)$ of $\lambda$-pseudo-starlike functions of order $\beta$ as follows:

Suppose $0 \leq \beta<1$ and $\lambda \geq 1$ is real. A function $f \in \mathcal{A}$ given by (1.1) belongs to the class $\mathscr{L}_{\lambda}(\beta)$ of $\lambda$-pseudo-starlike functions of order $\beta$ in the unit disk $\mathbb{U}$ if and only if

$$
\Re\left(\frac{z\left(f^{\prime}(z)\right)^{\lambda}}{f(z)}\right)>\beta \quad(z \in \mathbb{U}) .
$$

Babalola [1] proved that all pseudo-starlike functions are Bazilevič of type $1-1 / \lambda$, $\operatorname{order} \beta^{1 / \lambda}$ and univalent in $\mathbb{U}$.

Motivated by this definition, Joshi et al. [5] introduced the following two subclasses of the bi-univalent function class $\Sigma$ and obtained non-sharp estimates on the first two Taylor-Maclaurin coefficients $a_{2}$ and $a_{3}$ of functions in each of these subclasses.

Definition 1 ([5]). A function $f(z)$ given by (1.1) is said to be in the class $\mathscr{L} \mathscr{B}_{\Sigma}^{\lambda}(\alpha)$ if the following conditions are satisfied:

$$
f \in \Sigma \quad \text { and } \quad\left|\arg \left(\frac{z\left(f^{\prime}(z)\right)^{\lambda}}{f(z)}\right)\right|<\frac{\alpha \pi}{2} \quad(z \in \mathbb{U})
$$

and

$$
\left|\arg \left(\frac{w\left(g^{\prime}(w)\right)^{\lambda}}{g(w)}\right)\right|<\frac{\alpha \pi}{2} \quad(w \in \mathbb{U}),
$$

where $0<\alpha \leq 1, \lambda \geq 1$ and the function $g=f^{-1}$ is given by (1.2).

We call $\mathscr{L} \mathscr{B}_{\Sigma}^{\lambda}(\alpha)$ the class of strongly $\lambda$-bi-pseudo-starlike functions of order $\alpha$. Also for $\lambda=1$, we get $\mathscr{L} \mathscr{B}_{\Sigma}^{1}(\alpha)=\wp_{\Sigma}^{*}[\alpha]$ the class of strongly bi-starlike functions of order $\alpha$, introduced and studied by Brannan and Taha [2].

Theorem 1 ([5]). Let $f(z)$ given by (1.1) be in the class $\mathscr{L} \mathcal{B}_{\Sigma}^{\lambda}(\alpha)(0<\alpha \leq$ $1, \lambda \geq 1)$. Then

$$
\left|a_{2}\right| \leq \frac{2 \alpha}{\sqrt{(2 \lambda-1)(2 \lambda-1+\alpha)}}
$$


and

$$
\left|a_{3}\right| \leq \frac{4 \alpha^{2}}{(2 \lambda-1)^{2}}+\frac{2 \alpha}{3 \lambda-1} .
$$

Definition 2 ([5]). A function $f(z)$ given by (1.1) is said to be in the class $\mathscr{L} \mathscr{B}_{\Sigma}(\lambda, \beta)$ if the following conditions are satisfied:

$$
f \in \Sigma \quad \text { and } \quad \Re\left(\frac{z\left(f^{\prime}(z)\right)^{\lambda}}{f(z)}\right)>\beta \quad(z \in \mathbb{U})
$$

and

$$
\Re\left(\frac{w\left(g^{\prime}(w)\right)^{\lambda}}{g(w)}\right)>\beta \quad(w \in \mathbb{U}),
$$

where $0 \leq \beta<1, \lambda \geq 1$ and the function $g=f^{-1}$ is defined by (1.2).

We call $\mathscr{L} \mathscr{B}_{\Sigma}(\lambda, \beta)$ the class of $\lambda$-bi-pseudo-starlike functions of order $\beta$. Also for $\lambda=1$, we get $\mathscr{L} \mathscr{B}_{\Sigma}(1, \beta)=\mathcal{S}_{\Sigma}^{*}(\beta)$ the class of bi-starlike functions of order $\beta$, introduced and studied by Brannan and Taha [2].

Theorem 2 ([5]). Let $f(z)$ given by (1.1) be in the class $\mathscr{L} \mathscr{B}_{\Sigma}(\lambda, \beta)(0 \leq \beta<$ $1, \lambda \geq 1)$. Then

and

$$
\left|a_{2}\right| \leq \sqrt{\frac{2(1-\beta)}{\lambda(2 \lambda-1)}}
$$

$$
\left|a_{3}\right| \leq \frac{4(1-\beta)^{2}}{(2 \lambda-1)^{2}}+\frac{2(1-\beta)}{3 \lambda-1} .
$$

Here, in our present paper, inspiring by some of the aforecited works (especially [5]), we introduce the following subclass of the analytic function class $\mathcal{A}$, analogously to the definition given by $\mathrm{Xu}$ et al. [7].

Definition 3. Let the functions $h, p: \mathbb{U} \rightarrow \mathbb{C}$ be so constrained that

$$
\min \{\Re(h(z)), \Re(p(z))\}>0 \quad(z \in \mathbb{U}) \quad \text { and } \quad h(0)=p(0)=1 .
$$

Also let the function $f$ defined by (1.1) be in the analytic function class $\mathcal{A}$. We say that $f \in \mathscr{L}^{h, p}(\lambda)(\lambda \geq 1)$ if the following conditions are satisfied:

$$
f \in \Sigma \quad \text { and } \quad \frac{z\left(f^{\prime}(z)\right)^{\lambda}}{f(z)} \in h(\mathbb{U}) \quad(z \in \mathbb{U})
$$

and

$$
\frac{w\left(g^{\prime}(w)\right)^{\lambda}}{g(w)} \in p(\mathbb{U}) \quad(w \in \mathbb{U}),
$$

where the function $g=f^{-1}$ is defined by (1.2). 
Setting $\lambda=1$ in Definition 3, we get the class $\mathscr{L} \mathcal{B}_{\Sigma}^{h, p}(1)=\mathscr{B}_{\Sigma}^{h, p}$ introduced and studied by Bulut [3].

Remark 1. There are many choices of the functions $h$ and $p$ which would provide interesting subclasses of the analytic function class $\mathcal{A}$. For example, if we let

$$
h(z)=\left(\frac{1+z}{1-z}\right)^{\alpha} \quad \text { and } \quad p(z)=\left(\frac{1-z}{1+z}\right)^{\alpha} \quad(0<\alpha \leq 1, z \in \mathbb{U})
$$

or

$$
h(z)=\frac{1+(1-2 \beta) z}{1-z} \quad \text { and } \quad p(z)=\frac{1-(1-2 \beta) z}{1+z} \quad(0 \leq \beta<1, z \in \mathbb{U}),
$$

it is easy to verify that the functions $h(z)$ and $p(z)$ satisfy the hypotheses of Definition 3. If $f \in \mathscr{L}_{\Sigma}^{h, p}(\lambda)$, then

$$
f \in \Sigma \quad \text { and } \quad\left|\arg \left(\frac{z\left(f^{\prime}(z)\right)^{\lambda}}{f(z)}\right)\right|<\frac{\alpha \pi}{2} \quad(0<\alpha \leq 1, \lambda \geq 1, z \in \mathbb{U})
$$

and

$$
\left|\arg \left(\frac{w\left(g^{\prime}(w)\right)^{\lambda}}{g(w)}\right)\right|<\frac{\alpha \pi}{2} \quad(0<\alpha \leq 1, \lambda \geq 1, w \in \mathbb{U})
$$

or

$$
f \in \Sigma \quad \text { and } \quad \Re\left(\frac{z\left(f^{\prime}(z)\right)^{\lambda}}{f(z)}\right)>\beta \quad(0 \leq \beta<1, \lambda \geq 1, z \in \mathbb{U})
$$

and

$$
\Re\left(\frac{w\left(g^{\prime}(w)\right)^{\lambda}}{g(w)}\right)>\beta \quad(0 \leq \beta<1, \lambda \geq 1, w \in \mathbb{U}),
$$

where the function $g=f^{-1}$ is defined by (1.2). This means that

$$
f \in \mathscr{L} \mathcal{B}_{\Sigma}^{\lambda}(\alpha) \quad(0<\alpha \leq 1, \lambda \geq 1)
$$

or

$$
f \in \mathscr{L} \mathscr{B}_{\Sigma}(\lambda, \beta) \quad(0 \leq \beta<1, \lambda \geq 1) .
$$

Motivated and stimulated especially by the work of Joshi et al. [5], we propose to investigate the bi-univalent function class $\mathscr{L}_{B_{\Sigma}^{h, p}}^{h}(\lambda)$ introduced in Definition 3 here and derive coefficient estimates on the first two Taylor-Maclaurin coefficients $a_{2}$ and $a_{3}$ for a function $f \in \mathscr{L}^{h, p}(\lambda)$ given by (1.1). Our results for the bi-univalent function class $\mathscr{L} \mathscr{B}_{\Sigma}^{h, p}(\lambda)$ would generalize and improve the related work of Joshi et al. [5]. 


\section{A SET OF GENERAL COEFFICIENT ESTIMATES}

In this section we state and prove our general results involving the bi-univalent function class $\mathscr{L} \mathcal{B}_{\Sigma}^{h, p}(\lambda)$ given by Definition 3.

Theorem 3. Let the function $f(z)$ given by the Taylor-Maclaurin series expansion (1.1) be in the bi-univalent function class $\mathscr{L} \mathcal{B}_{\Sigma}^{h, p}(\lambda)$. Then

$$
\left|a_{2}\right| \leq \min \left\{\sqrt{\frac{\left|h^{\prime}(0)\right|^{2}+\left|p^{\prime}(0)\right|^{2}}{2(2 \lambda-1)^{2}}}, \sqrt{\frac{\left|h^{\prime \prime}(0)\right|+\left|p^{\prime \prime}(0)\right|}{4 \lambda(2 \lambda-1)}}\right\}
$$

and

where

$$
\left|a_{3}\right| \leq \min \{\gamma, \delta\}
$$

and

$$
\gamma=\frac{\left|h^{\prime}(0)\right|^{2}+\left|p^{\prime}(0)\right|^{2}}{2(2 \lambda-1)^{2}}+\frac{\left|h^{\prime \prime}(0)\right|+\left|p^{\prime \prime}(0)\right|}{4(3 \lambda-1)}
$$

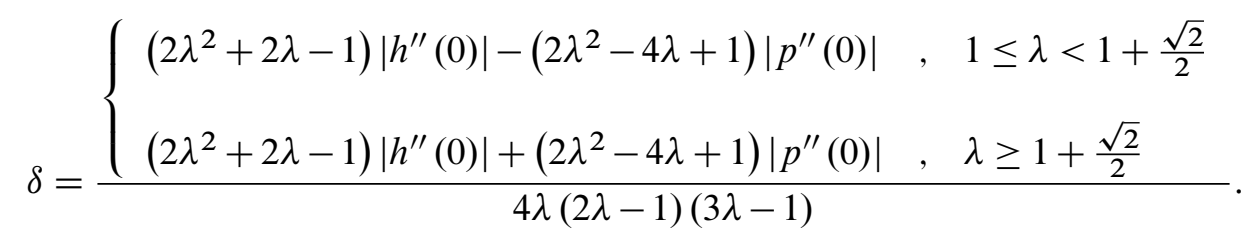

Proof. First of all, we write the argument inequalities in (1.11) and (1.12) in their equivalent forms as follows:

$$
\frac{z\left(f^{\prime}(z)\right)^{\lambda}}{f(z)}=h(z) \quad(z \in \mathbb{U})
$$

and

$$
\frac{w\left(g^{\prime}(w)\right)^{\lambda}}{g(w)}=p(w) \quad(w \in \mathbb{U}),
$$

respectively, where $h(z)$ and $p(w)$ satisfy the conditions of Definition 3. Furthermore, the functions $h(z)$ and $p(w)$ have the following Taylor-Maclaurin series expensions:

$$
h(z)=1+h_{1} z+h_{2} z^{2}+\cdots
$$

and

$$
p(w)=1+p_{1} w+p_{2} w^{2}+\cdots,
$$

respectively. Now, upon equating the coefficients of $\frac{z\left(f^{\prime}(z)\right)^{\lambda}}{f(z)}$ with those of $h(z)$ and the coefficients of $\frac{w\left(g^{\prime}(w)\right)^{\lambda}}{g(w)}$ with those of $p(w)$, we get

$$
\begin{gathered}
(2 \lambda-1) a_{2}=h_{1}, \\
\left(2 \lambda^{2}-4 \lambda+1\right) a_{2}^{2}+(3 \lambda-1) a_{3}=h_{2},
\end{gathered}
$$




$$
-(2 \lambda-1) a_{2}=p_{1}
$$

and

$$
\left(2 \lambda^{2}+2 \lambda-1\right) a_{2}^{2}-(3 \lambda-1) a_{3}=p_{2} .
$$

From (2.3) and (2.5), we obtain

$$
h_{1}=-p_{1}
$$

and

$$
2(2 \lambda-1)^{2} a_{2}^{2}=h_{1}^{2}+p_{1}^{2} .
$$

Also, from (2.4) and (2.6), we find that

$$
2 \lambda(2 \lambda-1) a_{2}^{2}=h_{2}+p_{2} .
$$

Therefore, we find from the equations (2.8) and (2.9) that

$$
\left|a_{2}\right|^{2} \leq \frac{\left|h^{\prime}(0)\right|^{2}+\left|p^{\prime}(0)\right|^{2}}{2(2 \lambda-1)^{2}}
$$

and

$$
\left|a_{2}\right|^{2} \leq \frac{\left|h^{\prime \prime}(0)\right|+\left|p^{\prime \prime}(0)\right|}{4 \lambda(2 \lambda-1)},
$$

respectively. So we get the desired estimate on the coefficient $\left|a_{2}\right|$ as asserted in (2.1).

Next, in order to find the bound on the coefficient $\left|a_{3}\right|$, we subtract (2.6) from (2.4). We thus get

$$
2(3 \lambda-1) a_{3}-2(3 \lambda-1) a_{2}^{2}=h_{2}-p_{2} .
$$

Upon substituting the value of $a_{2}^{2}$ from (2.8) into (2.10), it follows that

$$
a_{3}=\frac{h_{1}^{2}+p_{1}^{2}}{2(2 \lambda-1)^{2}}+\frac{h_{2}-p_{2}}{2(3 \lambda-1)} .
$$

We thus find that

$$
\left|a_{3}\right| \leq \frac{\left|h^{\prime}(0)\right|^{2}+\left|p^{\prime}(0)\right|^{2}}{2(2 \lambda-1)^{2}}+\frac{\left|h^{\prime \prime}(0)\right|+\left|p^{\prime \prime}(0)\right|}{4(3 \lambda-1)} .
$$

On the other hand, upon substituting the value of $a_{2}^{2}$ from (2.9) into (2.10), it follows that

$$
a_{3}=\frac{\left(2 \lambda^{2}+2 \lambda-1\right) h_{2}+\left(-2 \lambda^{2}+4 \lambda-1\right) p_{2}}{2 \lambda(2 \lambda-1)(3 \lambda-1)} .
$$

We thus obtain

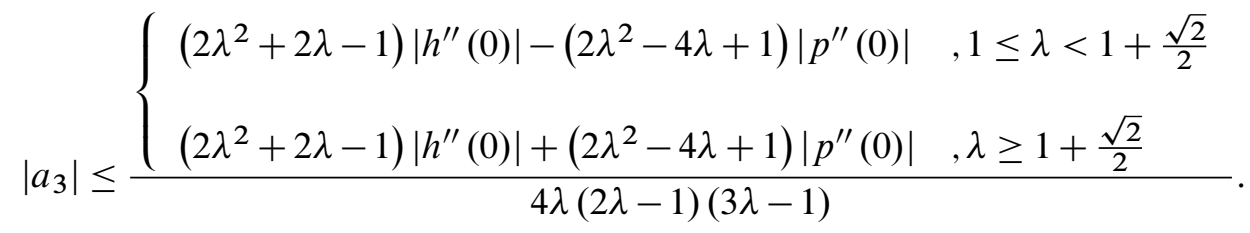


This evidently completes the proof of Theorem 3 .

\section{Corollaries AND CONSEQUences}

Setting $\lambda=1$ in Theorem 3, we get following consequence.

Corollary 1 ([3]). Let the function $f(z)$ given by the Taylor-Maclaurin series expansion (1.1) be in the bi-univalent function class $\mathcal{B}_{\Sigma}^{h, p}$. Then

$$
\left|a_{2}\right| \leq \min \left\{\sqrt{\frac{\left|h^{\prime}(0)\right|^{2}+\left|p^{\prime}(0)\right|^{2}}{2}}, \sqrt{\frac{\left|h^{\prime \prime}(0)\right|+\left|p^{\prime \prime}(0)\right|}{4}}\right\}
$$

and

$$
\left|a_{3}\right| \leq \min \left\{\frac{\left|h^{\prime}(0)\right|^{2}+\left|p^{\prime}(0)\right|^{2}}{2}+\frac{\left|h^{\prime \prime}(0)\right|+\left|p^{\prime \prime}(0)\right|}{8}, \frac{3\left|h^{\prime \prime}(0)\right|+\left|p^{\prime \prime}(0)\right|}{8}\right\} .
$$

If we set

$$
h(z)=\left(\frac{1+z}{1-z}\right)^{\alpha} \quad \text { and } \quad p(z)=\left(\frac{1-z}{1+z}\right)^{\alpha} \quad(0<\alpha \leq 1, z \in \mathbb{U})
$$

in Theorem 3, we can readily deduce Corollary 2 .

Corollary 2. Let the function $f(z)$ given by the Taylor-Maclaurin series expansion (1.1) be in the bi-univalent function class $\mathscr{L} \mathcal{B}_{\Sigma}^{\lambda}(\alpha)(0<\alpha \leq 1, \lambda \geq 1)$. Then

$$
\left|a_{2}\right| \leq \sqrt{\frac{2 \alpha^{2}}{\lambda(2 \lambda-1)}}
$$

and

$$
\left|a_{3}\right| \leq\left\{\begin{array}{cc}
\frac{2 \alpha^{2}}{\lambda(2 \lambda-1)} & , \quad 1 \leq \lambda<1+\frac{\sqrt{2}}{2} \\
\frac{2 \alpha^{2}}{3 \lambda-1} & \lambda \geq 1+\frac{\sqrt{2}}{2}
\end{array} .\right.
$$

Remark 2. Corollary 2 is an improvement of Theorem 1 .

If we set

$$
h(z)=\frac{1+(1-2 \beta) z}{1-z} \quad \text { and } \quad p(z)=\frac{1-(1-2 \beta) z}{1+z} \quad(0 \leq \beta<1, z \in \mathbb{U})
$$

in Theorem 3, we can readily deduce Corollary 3 .

Corollary 3. Let the function $f(z)$ given by the Taylor-Maclaurin series expansion (1.1) be in the bi-univalent function class $\mathscr{L} \mathcal{B}_{\Sigma}(\lambda, \beta)(0 \leq \beta<1, \lambda \geq 1)$. Then

$$
\left|a_{2}\right| \leq\left\{\begin{array}{cc}
\sqrt{\frac{1-\beta}{\lambda(2 \lambda-1)}} & 0 \leq \beta \leq \frac{2 \lambda+1}{4 \lambda} \\
\frac{2(1-\beta)}{(2 \lambda-1)} & \frac{2 \lambda+1}{4 \lambda} \leq \beta<1
\end{array}\right.
$$


and

$$
\left|a_{3}\right| \leq\left\{\begin{array}{cc}
\min \left\{\frac{4(1-\beta)^{2}}{(2 \lambda-1)^{2}}+\frac{1-\beta}{3 \lambda-1}, \frac{1-\beta}{\lambda(2 \lambda-1)}\right\} & 1 \leq \lambda<1+\frac{\sqrt{2}}{2} \\
\frac{1-\beta}{3 \lambda-1} & \lambda \geq 1+\frac{\sqrt{2}}{2}
\end{array} .\right.
$$

Remark 3. Corollary 3 is an improvement of Theorem 2.

\section{REFERENCES}

[1] K. Babalola, “On $\lambda$-pseudo-starlike functions,” J. Class. Anal., vol. 3, no. 2, pp. 137-147, 2013, doi: $10.7153 /$ jca-03-12.

[2] D. Brannan and T. Taha, "On some classes of bi-univalent functions," Studia Univ. Babeş -Bolyai Math., vol. 31, no. 2, pp. 70-77, 1986, doi: 10.1016/B978-0-08-031636-9.50012-7.

[3] S. Bulut, "Coefficient estimates for a class of analytic and bi-univalent functions," Novi Sad J. Math., vol. 43, no. 2, pp. 59-65, 2013.

[4] P. Duren, Univalent Functions, ser. Grundlehren der Mathematischen Wissenschaften. New York: Springer, 1983, vol. 259.

[5] S. Joshi, S. Joshi, and H. Pawar, "On some subclasses of bi-univalent functions associated with pseudo-starlike functions," J. Egyptian Math Soc., vol. in press, doi: 10.1016/j.joems.2016.03.007.

[6] H. Srivastava, A. Mishra, and P. Gochhayat, "Certain subclasses of analytic and bi-univalent functions," Appl. Math. Lett., vol. 23, pp. 1188-1192, 2010, doi: 10.1016/j.aml.2010.05.009.

[7] Q.-H. Xu, Y.-C. Gui, and H. Srivastava, "Coefficient estimates for a certain subclass of analytic and bi-univalent functions," Appl. Math. Lett., vol. 25, pp. 990-994, 2012, doi: 10.1016/j.aml.2011.11.013.

[8] Q.-H. Xu, H.-G. Xiao, and H. Srivastava, "A certain general subclass of analytic and bi-univalent functions and associated coefficient estimate problems," Appl. Math. Comput., vol. 218, pp. $11461-$ 11 465, 2012, doi: 10.1016/j.amc.2012.05.034.

\section{Author's address}

\section{Serap Bulut}

Kocaeli University, Faculty of Aviation and Space Sciences, Arslanbey Campus, 41285 KartepeKocaeli, Turkey

E-mail address: serap.bulut@kocaeli.edu.tr 